\title{
A Distributed Observer for a Time-Invariant Linear System
}

\author{
L. Wang ${ }^{1}$, and A. S. Morse ${ }^{1}$
}

\begin{abstract}
A time-invariant, linear, distributed observer is described for estimating the state of an $m>0$ channel, $n$ dimensional continuous-time linear system of the form $\dot{x}=$ $A x, y_{i}=C_{i} x, i \in\{1,2, \ldots, m\}$. The state $x$ is simultaneously estimated by $m$ agents assuming each agent $i$ senses $y_{i}$ and receives the state $z_{j}$ of each of its neighbors' estimators. Neighbor relations are characterized by a constant directed graph $\mathbb{N}$ whose vertices correspond to agents and whose arcs depict neighbor relations. For the case when the neighbor graph is strongly connected, the overall distributed observer consists of $m$ linear estimators, one for each agent; $m-1$ of the estimators are of dimension $n$ and one estimator is of dimension $n+m-1$. Using results from classical decentralized control theory, it is shown that subject to the assumptions that (i) none of the $C_{i}$ are zero, (ii) the neighbor graph $\mathbb{N}$ is strongly connected, (iii) the system whose state is to be estimated is jointly observable, and nothing more, it is possible to freely assign the spectrum of the overall distributed observer. For the more general case when $\mathbb{N}$ has $q>1$ strongly connected components, it is explained how to construct a family of $q$ distributed observers, one for each component, which can estimate $x$ at a preassigned convergence rate.
\end{abstract}

\section{INTRODUCTION}

State estimators such as Kalman filters and observers have had a huge impact on the entire field of estimation and control. This paper deals with observers for time-invariant linear systems. An observer for a process modeled by a continuous-time, time-invariant linear system with state $x$, measured output $y=C x$ and state-dynamics $\dot{x}=A x$, is a time-invariant linear system with input $y$ which is capable of generating an asymptotically correct estimate of $x$ exponentially fast at a pre-assigned but arbitrarily large convergence rate. As is well known, the only requirement on the system $y=C x, \dot{x}=A x$ for such an estimator to exist is that the matrix pair $(C, A)$ be observable. In this paper we will be interested in the natural generalization of this concept appropriate to a network of $m$ agent. We now make precise what we mean by this.

\section{A. The Problem}

We are interested in a fixed network of $m>0$ autonomous agents labeled $1,2, \ldots, m$ which are able to receive information from their neighbors where by the neighbor of agent $i$ is meant any other agent in agent $i$ 's reception range. We write $\mathcal{N}_{i}$ for the set of labels of agent $i$ 's neighbors and we take agent $i$ to be a neighbor of itself. Neighbor relations between

*This work was supported by National Science Foundation grant $\mathrm{n}$. 1607101.00 and US Air Force grant n. FA9550-16-1-0290.

1 L. Wang and A. S. Morse are with the Department of Electrical Engineering, Yale University, New Haven, CT, USA. \{lili.wang, as.morse\}@yale.edu distinct pairs of agents are characterized by a directed graph $\mathbb{N}$ with $m$ vertices and a set of arcs defined so that there is an arc from vertex $j$ to vertex $i$ if whenever agent $j$ is a distinct neighbor of agent $i$. Each agent $i$ can sense a signal $y_{i} \in \mathbb{R}^{s_{i}}, i \in \mathbf{m}=\{1,2, \ldots, m\}$, where

$$
\begin{aligned}
y_{i} & =C_{i} x, \quad i \in \mathbf{m} \\
\dot{x} & =A x
\end{aligned}
$$

and $x \in \mathbb{R}^{n}$.

Agent $i$ estimates $x$ using an $n_{i}$ dimensional linear system with state vector $z_{i}$ and we assume the information agent $i$ can receive from neighbor $j \in \mathcal{N}_{i}$ is $z_{j}(t)$ and $y_{j}(t)$. The problem of interest is to construct a suitably defined family of linear systems

$$
\begin{aligned}
\dot{z}_{i} & =\sum_{j \in \mathcal{N}_{i}}\left(H_{i j} z_{j}+K_{i j} y_{j}\right), \quad i \in \mathbf{m} \\
x_{i} & =\sum_{j \in \mathcal{N}_{i}}\left(M_{i j} z_{j}+N_{i j} y_{j}\right), \quad i \in \mathbf{m}
\end{aligned}
$$

in such a way so that no matter what the initializations of (1) and (3), each signal $x_{i}(t)$ is an asymptotically correct estimate of $x(t)$ in the sense that each estimation error $e_{i}=x_{i}(t)-x(t)$ converges to zero as $t \rightarrow \infty$ at a preassigned, but arbitrarily fast convergence rate. We call such a family a distributed (state) observer.

We assume throughout that $C_{i} \neq 0, i \in \mathbf{m}$, and that the system defined by (1), (2) is jointly observable; i.e., with $C=\left[\begin{array}{llll}C_{1}^{\prime} & C_{2}^{\prime} & \cdots & C_{m}^{\prime}\end{array}\right]^{\prime}$, the matrix pair $(C, A)$ is observable. Generalizing the results which follow to the case when $(C, A)$ is only detectable is quite straightforward and can be accomplished using well-known ideas. However the commonly made assumption that each pair $\left(C_{i}, A\right), i \in \mathbf{m}$, is observable, or even just detectable, is very restrictive, grossly simplifies the problem and is as unnecessary. It is precisely the exclusion of this assumption which distinguished the problem posed here from almost all of the distributed estimator problems addressed in the literature. The one exception we are aware of is the recent paper [1] which has provided the main motivation for this work.

\section{B. Background}

There is a huge literature which seeks to deal with distributed Kalman filters or distributed observers; see, for example [1]-[6] and the many references cited therein. Many result are only partial and most problem formulations are different in detail than the problem posed here. The problem we have posed was prompted specifically by the work in [1] which 
seeks to devise a time-invariant distributed observer for the discrete-time analog of (1), (2). Two particularly important contributions are made in [1]. First it is recognized that the problem of crafting a 'stable' distributed observer is more or less equivalent to devising a stabilizing decentralized control as in [7], [8]. Second, it is demonstrated that under suitable conditions, it is only necessary for the dimension of one of the agent subsystems in [1] to be larger than $n$, and that the enlarged dimension need not exceed $n+m-1$.

The work reported in this paper clarifies and expand on the results of [1] in several ways. First we outline a construction for systems with strongly connected neighbor graphs which enables one to freely adjust the observer's spectrum. Second, the results obtained here apply whether $A$ is singular or not; the implication of this generalization is that the construction proposed can be used to craft observers for continuous time processes whereas the construction proposed in [1] cannot unless $A$ is nonsingular.

\section{OBSERVER DESIGN EQUATIONS}

We now develop the interrelationships between the matrices appearing in (3) and (4) which must hold for each $x_{i}$ to be an asymptotically correct estimate of $x$. Note first that because (4) must hold even when all estimates are correct, for each $i \in \mathbf{m}$ it is necessary that the equation $x=\sum_{j \in \mathcal{N}_{i}}\left(M_{i j} z_{j}+N_{i j} C_{j} x\right), \quad i \in \mathbf{m}$ have a solution $z_{i}^{x}, \quad i \in \mathbf{m}$, for each possible $x \in \mathbb{R}^{n}$. Thus if we define $V_{i}=\left[\begin{array}{llll}z_{i}^{u_{1}} & z_{i}^{u_{2}} & \cdots & z_{i}^{u_{n}}\end{array}\right]_{n_{i} \times n}, i \in \mathbf{m}$, where $u_{k}$ is the $k$ th unit vector in $\mathbb{R}^{n}$, then

$$
I=\sum_{j \in \mathcal{N}_{i}}\left(M_{i j} V_{j}+N_{i j} C_{j}\right), \quad i \in \mathbf{m}
$$

This and (4) imply that the $m$ estimation errors satisfy

$$
x_{i}-x=\sum_{j \in \mathcal{N}_{i}} M_{i j} \epsilon_{j}, \quad i \in \mathbf{m}
$$

where

$$
\epsilon_{i}=z_{i}-V_{i} x, i \in \mathbf{m}
$$

Moreover, as a direct consequence of (1), (2), and (3),

$\dot{\epsilon}_{i}=\sum_{j \in \mathcal{N}_{i}} H_{i j} \epsilon_{j}+\left(\sum_{j \in \mathcal{N}_{i}}\left(H_{i j} V_{j}+K_{i j} C_{j}\right)-V_{i} A\right) x, \quad i \in \mathbf{m}$

Thus if we stipulate that

$$
V_{i} A=\sum_{j \in \mathcal{N}_{i}}\left(H_{i j} V_{j}+K_{i j} C_{j}\right), i \in \mathbf{m}
$$

then

$$
\dot{\epsilon}_{i}=\sum_{j \in \mathcal{N}_{i}} H_{i j} \epsilon_{j}, i \in \mathbf{m}
$$

We shall refer to (5) and (8) as the observer design equations. These equations are quite general. They apply to all timeinvariant continuous and discrete time state observers whether they are distributed or not.

It is clear from (6) that if the $V_{i}, H_{i j}, M_{i j}, N_{i j}$ and $K_{i j}$ can be chosen so that the observer design equations (5), (8) hold and the system defined by 9 is exponentially stable, then each $x_{i}$ will be an asymptotically correct estimate of $x$. The distributed observer design problem is to develop constructive conditions which ensure that the $V_{i}, H_{i j}, M_{i j}, N_{i j}$ and $K_{i j}$ can be so chosen.

\section{Centralized Observers}

The purpose of this section is to review the well-known concept of a (centralized) observer with the aim summarizing certain less well know ideas which will play a role in the construction of a distributed observer. In the centralized case $m=1$ and a state observer is a $n_{1}$-dimensional linear system with input $y=C x$, state $z \in \mathbb{R}^{n_{1}}$ and output $x_{1}$ of the form $\dot{z}=H z+K y, x_{1}=M z+N y$. In this case the observer design equations are $I=M V+N C$ and $V A=H V+K C$ and the observer design problem is to determine matrices $H, K, M, N$ and $V$ so that the observer design equations hold and $H$ is a stability matrix. Observers fall into three broad categories depending on the dimension $n_{1}$ : full state observers, minimal state observers, and extended state observers. Each type is briefly reviewed below.

Full-State Observers: Just about the easiest solution to the observer design problem that one can think of, is the one for which $M \triangleq I, N=0, V=I_{n \times n}$ and $H \triangleq A-K C$. Any observer of this type is called a full-state observer because in this case $z_{1}$ is an asymptotic estimate of $x$. Of course it is necessary that $K$ be chosen so that $A-K C$ is a stable matrix. One way to accomplish this is to exploit duality and use spectrum assignment, as is well known. No matter how one goes about defining $K$, the definitions of $H, M, N$, and $V$ given above show that a full-state observer is modeled by equations of the form $\dot{z}_{1}=(A-K C) z_{1}+K y, x_{1}=z_{1}$.

Reduced State Observers: By a minimal state observer is meant an observer of least dimension which can generate an asymptotic estimate of $x$. Minimal dimensional observers are obtained by exploiting the fact that $y=C x$ is a "partial" measurement of $x$. Note that the observer design equation $I=M V+N C$ implies that the number of linearly independent rows of $V_{n_{1} \times n}$ must be at least equal to the dimension of $\operatorname{ker} C$. Thus the dimension of any observer must be at least equal to dimension $\operatorname{ker} C$. Techniques for constructing minimal state observers are well known [9], [10].

Extended State Observers: Much less well known are what might be called 'extended state observers.' A observer of this type would be of dimension $n_{1}=n+\bar{n}$ where $\bar{n}$ is a nonnegative integer chosen by the designer. With $\bar{n}$ fixed, an extended observer can be obtained by first picking $M=\left[\begin{array}{ll}I & 0\end{array}\right]_{n \times(n+\bar{n})}, V^{\prime}=\left[\begin{array}{ll}I & 0\end{array}\right]_{n \times(n+\bar{n})}$ and $N=0$ thereby ensuring that observer design equation $I=M V+N C$ is satisfied. With $V$ so chosen, $z_{1}$ must be of the corresponding form $z_{1}=\left[\begin{array}{ll}x_{1}^{\prime} & \bar{z}_{1}^{\prime}\end{array}\right]^{\prime}$. Accordingly, the partitioned matrices

$$
H=\left[\begin{array}{cc}
A+\bar{D} C & \bar{C} \\
\bar{B} C & \bar{A}
\end{array}\right]_{(n+\bar{n}) \times(n+\bar{n})} \quad K=-\left[\begin{array}{c}
\bar{D} \\
\bar{B}
\end{array}\right]
$$

satisfy the observer design equation $V A=H V+K C$ for any 
values of the matrices $\bar{A}, \bar{B}, \bar{C}, \bar{D}$ and

$$
\begin{aligned}
\dot{x}_{1} & =(A+\bar{D} C) x_{1}+\bar{C} \bar{z}_{1}-\bar{D} y \\
\dot{\bar{z}}_{1} & =\bar{B} C x_{1}+\bar{A} \bar{z}_{1}-\bar{B} y
\end{aligned}
$$

Moreover the estimation error $e=x_{1}-x$ satisfies

$$
\begin{aligned}
\dot{e} & =(A+\bar{D} C) e+\bar{C} \bar{z}_{1} \\
\dot{\bar{z}}_{1} & =\bar{B} C e+\bar{A} \bar{z}_{1}
\end{aligned}
$$

These equations suggests the following feedback diagram.

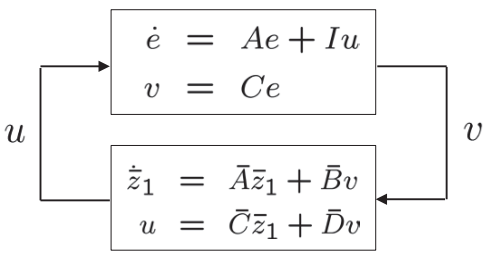

Thus the design of an extended state observer amounts to picking the coefficient matrices $\{\bar{A}, \bar{B}, \bar{C}, \bar{D}\}$ of the lower subsystem in the block diagram to at least stabilize the loop. Of course if $\bar{n}=0$, this subsystem is just the constant matrix $\bar{D}$ and one has again a classical full-state observer of dimension $n$. Exactly what might be gained by picking $\bar{n}$ greater than zero is not clear in the case of a centralized observer. However, for the decentralized observer we describe next, the flexibility of a dynamic lower loop will become self-evident.

\section{Distributed OBSERVERS}

The primary goal of distributed observer design is to choose the matrices $V_{i}, H_{i j}, M_{i j}, N_{i j}$ and $K_{i j}$ so that the observer design equations (5) and (8) hold and the system defined by (9) is exponentially stable. Another goal might be to choose these matrices to reduce the information which needs to be transmitted between neighboring agents. Still another goal might be to choose these matrices so that the dimensions of the individual estimators are as small as possible. In this paper we will consider the case when the only information transmitted between neighboring agents are estimator states $z_{i}$ and we will make no attempt to construct estimators of least dimension. This means that we will set all $K_{i j}=0$ except for $K_{i i}$ in (3) and all $N_{i j}=0$ in (4). The easiest way to satisfy the observer design equations is to set $V_{i}=I_{n \times n}$ for $i \in \mathbf{m}$ and to pick the $M_{i j}$ so that $I=\sum_{j \in \mathcal{N}_{i}} M_{i j}, \quad i \in \mathbf{m}$. With the $V_{i}$ so chosen, observer design equation (8) simplifies to

$$
A-K_{i} C_{i}=\sum_{j \in \mathcal{N}_{i}} H_{i j}, i \in \mathbf{m}
$$

where we have adopted the notation $K_{i}=K_{i i}$ In view of (6) and (9), the observer design problem for this type of an observer is to try to choose the $K_{i}$ and $H_{i j}$ so that holds and in addition so that $H=\left[H_{i j}\right]$ is a stability matrix where $H_{i j}=0$ if $j \notin \mathcal{N}_{i}$. It is possible to express $H$ in a more explicit form which takes into account the constraints on the $H_{i j}$ imposed by (10). For this let $\tilde{A}$ denote the block diagonal matrix $\tilde{A}=I_{m \times m} \otimes A$ where $\otimes$ is the Kronecker product. Set $B_{i}=b_{i} \otimes I_{n \times n} i \in \mathbf{m}$ where $b_{i}$ is the $i$ th unit vector in $\mathbb{R}^{m}$; in addition, let $C_{i i}=C_{i} B_{i}^{\prime}, i \in \mathbf{m}$, and $C_{i j}=c_{i j} \otimes I_{n \times n}, \quad j \in$ $\mathcal{N}_{i}, j \neq i, i \in \mathbf{m}$ where $c_{i j}$ is the row in the transpose of the incidence matrix of $\mathbb{N}$ corresponding to the arc from $j$ to $i$. It is then possible to express $H$ in the compact form

$$
H=\tilde{A}+\sum_{i \in \mathbf{m}} \sum_{j \in \mathcal{N}_{i}} B_{i} F_{i j} C_{i j}
$$

where $F_{i i}=-K_{i}, i \in \mathbf{m}$ and $F_{i j}=H_{i j}, j \in \mathcal{N}_{i}, j \neq i, i \in$ m. Note that there are no constraints on the $F_{i j}$. In this form it is clear that $H$ is what results when output feedback laws $u_{i j}=F_{i j} y_{i j}$ are applied to the system

$$
\begin{aligned}
\dot{\epsilon} & =\tilde{A} \epsilon+\sum_{i \in \mathbf{m}} \sum_{j \in \mathcal{N}_{i}} B_{i} u_{i j} \\
y_{i j} & =C_{i j} \epsilon, \quad i j \in \mathcal{I}
\end{aligned}
$$

where $\mathcal{I} \subset \mathbf{m} \times \mathbf{m}$ is the set of double indices $\mathcal{I}=\{i j$ : $\left.i \in \mathbf{m}, j \in \mathcal{N}_{i}\right\}$. The problem of constructing a distributed observer of this type thus reduces to trying to choose the $F_{i j}$ to at least stabilize $H$ if such matrices exist. Of course, one also wants control over rate of convergence, so stabilization of $H$ alone is not all that is of interest. Whether the goal is just stabilization of $H$ or control over convergence rate, choosing the $F_{i j}$ to accomplish this will typically not be possible except under special conditions. In fact the problem trying to stabilize $H$ by appropriately choosing the $F_{i j}$ is mathematically the same as the classical decentralized stabilization problem for which there is a substantial literature [7], [8].

\section{A. Strongly Connected Neighbor Graph $\mathbb{N}$}

One approach is to decentralized stabilization problem is to try to choose the $F_{i j}$ so that for given $p \in \mathbf{m}$ and $q \in \mathcal{N}_{p}$, the matrix pairs $\left(H, B_{p}\right)$ and $\left(C_{p q}, H\right)$ are controllable and observable respectively. Having accomplished this, stabilization can then be achieved by applying standard centralized feedback techniques such as those in [11] to the resulting controllable observable system. This is the approach taken in this paper. The following proposition provides the key technical result which we need.

Proposition 1: Suppose that the neighbor graph $\mathbb{N}$ is strongly connected. There exist gain matrices $F_{i j}, i j \in \mathcal{I}$ such that the matrix pairs $\left(H, B_{p}\right)$ and $\left(C_{p q}, H\right)$ are controllable and observable respectively for all $p \in \mathbf{m}$ and all $q \in \mathcal{N}_{p}$. Moreover, for any such pair, $m$ is the controllability index of $\left(H, B_{p}\right)$.

The proof of this proposition will be given is Section VI

In the light of Proposition 11 the way to construct a distributed observer is clear. As a first step, choose matrices $M_{i j}, i \in \mathbf{m}, j \in \mathcal{N}_{i}$ so that $I=\sum_{j \in \mathcal{N}_{i}} M_{i j}, i \in \mathbf{m}$. Next choose the $F_{p q}$ so that the conclusions of the Proposition 1 hold. Having so chosen the $F_{i j}$ or equivalently the $H_{i j}$ and the $K_{i}$, fix values of $p \in \mathbf{m}$ and $q \in \mathcal{N}_{p}$. Next set $\bar{n}=m-1$ and use a standard construction technique such as that given in [11] to pick matrices $\bar{A}_{\bar{n} \times \bar{n}}, \bar{B}_{\bar{n} \times \omega}, \bar{C}_{n \times \bar{n}}$ and $\bar{D}_{n \times \omega}$ to assign a desirable spectrum to the matrix

$$
\bar{H}=\left[\begin{array}{cc}
H+B_{p} \bar{D} C_{p q} & B_{p} \bar{C} \\
\bar{B} C_{p q} & \bar{A}
\end{array}\right]_{(n m+\bar{n}) \times(n m+\bar{n})}
$$


where $\omega=s_{p}$ if $q=p$ or $\omega=n$ if $p \neq q$. This can be done because $\left(C_{p q}, H\right)$ is an observable pair and because $\left(H, B_{p}\right)$ is a controllable pair with controllability index $m$. The corresponding distributed observer equations are

$$
\begin{aligned}
\dot{z}_{i} & =\sum_{k \in \mathcal{N}_{i}} H_{i k} z_{k}+K_{i} y_{i}, \quad i \in \mathbf{m}, i \neq p \\
\dot{z}_{p} & =\sum_{k \in \mathcal{N}_{p}} H_{p k} z_{k}+K_{p} y_{p}+\bar{C} \bar{z}+\bar{D} v \\
\dot{\bar{z}} & =\bar{A} \bar{z}+\bar{B} v \\
x_{i} & =\sum_{k \in \mathcal{N}_{i}} M_{i k} z_{k}, \quad i \in \mathbf{m}
\end{aligned}
$$

where $v=y_{p}$ if $q=p$ or $v=x_{p}-x_{q}$ if $p \neq q$.

It is possible to verify that the observer design equations hold. For simplicity, assume that $p=m$ and redefine $V_{m}$ to be $\left[\begin{array}{ll}I & 0\end{array}\right]_{(n+\bar{n}) \times n}^{\prime}$. For $k \in \mathbf{m}$, redefine $M_{k m}$ to be $\left[\begin{array}{ll}M_{k m} & 0\end{array}\right]_{\omega \times(n+\bar{n})}$ thereby ensuring that observer design equation (5) holds. To ensure that observer design equation (8) holds, first replace $H_{k m}$ with $\left[\begin{array}{ll}H_{k m} & 0\end{array}\right]_{n \times(n+\bar{n})}$ for $k \in\{1,2, \ldots, m-1\}$. If $q=m$ replace $H_{m i}, i \in \mathbf{m}, i \neq m$, and $H_{m m}$ with the matrices

$$
\left[\begin{array}{c}
H_{m i} \\
0
\end{array}\right], i \in\{1,2, \ldots, m-1\}
$$

and

$$
\left[\begin{array}{cc}
H_{m m}+\bar{D} C_{m} & \bar{C} \\
\bar{B} C_{m} & \bar{A}
\end{array}\right]
$$

respectively; in addition, replace $K_{m}$ with the matrix $\left[\begin{array}{ll}\left(K_{m}-\bar{D}\right)^{\prime} & -\bar{B}^{\prime}\end{array}\right]_{(n+\bar{n}) \times s_{m}}^{\prime}$. If on the other hand, $m \neq q$, replace $H_{m i}, i \in \mathbf{m}, i \neq p, m, H_{m p}$ and $H_{m m}$ with the matrices

$$
\begin{gathered}
{\left[\begin{array}{c}
H_{m i} \\
0
\end{array}\right], i \in\{1,2, \ldots, m-1\}, i \neq q,} \\
{\left[\begin{array}{c}
H_{m q}-\bar{D} \\
-\bar{B}
\end{array}\right], \quad \text { and }\left[\begin{array}{cc}
H_{m m}+\bar{D} & \bar{C} \\
\bar{B} & \bar{A}
\end{array}\right]}
\end{gathered}
$$

respectively; in addition, replace $K_{m}$ with matrix $\left[\begin{array}{ll}K_{m}^{\prime} & 0\end{array}\right]_{(n+\bar{n}) \times s_{m}}^{\prime}$. In either case, observer design equation (8) holds. Thus the error $\bar{\epsilon}=\left[\begin{array}{lllll}\epsilon_{1}^{\prime} & \epsilon_{2}^{\prime} & \ldots & \epsilon_{m}^{\prime} & \bar{z}^{\prime}\end{array}\right]^{\prime}$ satisfies $\dot{\bar{\epsilon}}=\bar{H} \bar{\epsilon}$ where as before, $x_{p}-x=\sum_{k \in \mathcal{N}_{p}} M_{p k} \epsilon_{p}, \quad p \in \mathbf{m}$.

We are led to the main result of this paper.

Theorem 1: Suppose that (1), (2) is a jointly observable system and that $C_{i} \neq 0, i \in \mathbf{m}$. If the neighbor graph $\mathbb{N}$ is strongly connected, then for each symmetric set of $m n+m-1$ complex numbers $\Lambda$ there is a distributed observer (3), (4) for which the spectrum of the $(m n+m-1) \times(m n+m-1)$ matrix $H \triangleq\left[H_{i j}\right]$ is $\Lambda$. Moreover, the observer's $m$ outputs $x_{i}(t), i \in \mathbf{m}$, all asymptotically correctly estimate $x(t)$ in the sense that each estimation error $e_{i}=x_{i}(t)-x(t)$ converges to zero as $t \rightarrow \infty$ as fast $e^{H t}$ converges to zero, no matter what the initializations of (2) and (3) are.

\section{B. Non-Strongly Connected Neighbor Graph $\mathbb{N}$}

We now turn briefly to the problem of developing a distributed observer for the case when $\mathbb{N}$ is not strongly connected. We will assume for simplicity and without loss of generality that $\mathbb{N}$ is weakly connected. For if it is not, the ideas which follow can be applied to each maximally weakly connected subgraph of $\mathbb{N}$, since each such subgraph is isolated from the rest. As before, the goal is to devise $m$ estimators whose estimates converge to $x$ exponentially fast at arbitrary, pre-assigned rates. We suppose that $\mathbb{N}$ has $q$ strongly connected components $\mathbb{N}_{1}, \mathbb{N}_{2}, \ldots, \mathbb{N}_{q}$ and for each $i \in \mathbf{q}$ we write $\Sigma_{i}$ for the $m_{i}$ channel component subsystem $\dot{x}=A x$ $y_{j}=C_{j} x, j \in \mathcal{V}_{i}$ where $\mathcal{V}_{i}$ is the set of labels of the vertices of $\mathbb{N}_{i}$ and $m_{i}$ is the number of labels in $\mathcal{V}_{i}$. We say that there is a directed path $\{$ resp. arc $\}$ from strongly connected component $\mathbb{N}_{i}$ to strongly connected component $\mathbb{N}_{j}$ if there is a directed path $\{$ resp. arc $\}$ in $\mathbb{N}$ from at least one vertex in $\mathbb{N}_{i}$ to at least one vertex in $\mathbb{N}_{j}$. Following [1], we say that $\mathbb{N}_{j}$ is a source component of $\mathbb{N}$ if $\mathbb{N}_{j}$ has no incoming arcs from any other strongly connected component of $\mathbb{N}$. It is clear that $\mathbb{N}$ must contain at at least one source component. Moreover, since $\mathbb{N}$ is weakly connected, it is also clear that for any strongly connected component of $\mathbb{N}_{i}$ which is not a source, there must be at least one directed path from at least one source $\mathbb{N}_{j}$ to $\mathbb{N}_{i}$.

Let $\mathbb{N}_{j}$ be a source component and $\Sigma_{j}$ be its associated component subsystem. Note that there cannot be any signal flow to any channel in $\Sigma_{j}$ from any channel of any other component subsystem. It follows that for there to exist estimators for each channel in $\Sigma_{j}$ which are capable of estimating $x$ at a preassigned convergence rate, it is necessary that $\Sigma_{j}$ be a jointly observable subsystem. In view of Theorem 11 joint observability of $\Sigma_{j}$ is also sufficient for such a distributed observer to exist because $\mathbb{N}_{j}$ is strongly connected. Suppose therefore that for each source component $\mathbb{N}_{j}$, the associated component subsystem $\Sigma_{j}$ is jointly observable and that a distributed observer has been constructed with preassigned converge rate for each such $\Sigma_{j}$. If all strongly connected components of $\mathbb{N}$ are sources, then these observers solve the distribute observer design problem. Suppose therefore that there is at least one strongly connected component which is not a source. Then there must be at least one strongly connected component $\mathbb{N}_{i}$ which is not a source for which there is a source $\mathbb{N}_{j}$ with an arc to $\mathbb{N}_{i}$. This implies that there must be a channel $k \in \mathcal{V}_{j}$ of $\Sigma_{j}$ whose estimator state $z_{k}$ is available to at least one channel - say channel $l$ of component subsystem $\Sigma_{i}$. But $\epsilon_{k}=z_{k}-V_{k} x$. Moreover, for the full-state observers we are considering, $V_{k}^{\prime}$ is a left inverse of $V_{k}$ so $V_{k}^{\prime} z_{k}=\bar{C}_{l} x+V_{k}^{\prime} \epsilon_{k}$ where $\bar{C}_{l}=I_{n \times n}$. Therefore $V_{k}^{\prime} z_{k}$ can be regarded as a measurement of $x$ with exponentially decaying additive measurement noise $V_{k}^{\prime} \epsilon_{k}$. Thus if the readout equation $y_{l}=C_{l} x$ in the definition of $\Sigma_{i}$, is replaced with with the augmented readout equation

$$
y_{l}=\left[\begin{array}{c}
C_{l} \\
\bar{C}_{k}
\end{array}\right] x+\left[\begin{array}{c}
0 \\
V_{k}^{\prime} \epsilon_{k}
\end{array}\right],
$$

then the resulting subsystem, denoted by $\bar{\Sigma}_{i}$ will be jointly observable with unmeasurable but exponentially decaying 
measurement noise. Since $\mathbb{N}_{i}$ is strongly connected, a distributed observer with the same convergent rate as that of $\epsilon_{k}$, can therefore be constructed for $\Sigma_{i}$. If $\mathbb{N}_{i}$ is the only strongly connected component of $\mathbb{N}$ which is not a source, then construction is complete. If, on the other hand, $\mathbb{N}$ has other strongly connected components which are not sources, the same ideas as just described, can be applied to each corresponding component subsystem in a sequential manner. We are led to the following

Corollary 1: Suppose that $C_{i} \neq 0, i \in \mathbf{m}$ and that neighbor graph $\mathbb{N}$ has $q$ strongly connected components $\mathbb{N}_{i}, i \in \mathbf{q}$. Let $\Sigma_{i}$ be the component subsystem of (1), (2) corresponding to strongly connected component $i$. In order for there to exist distributed observers for each of the component subsystems which are a capable of estimating $x$ at an arbitrary but preassigned convergence rate, it is necessary and sufficient that each of the component subsystems whose graphs are sources, are jointly observable.

\section{Decentralized Control Theory}

The aim of this section is to summarize the concepts and results from [8] and [12] which we will make use of to justify Proposition 1 We do this for a $k$ channel, $n$-dimensional linear system of the form

$$
\dot{x}=A x+\sum_{i \in \mathcal{I}} B_{i} u_{i} \quad y_{i}=C_{i} x, \quad i \in \mathcal{I}
$$

where $\mathcal{I}=\{1,2, \ldots, k\}$ and $C_{i} \neq 0, \quad i \in \mathcal{I}$. Application of decentralized feedback laws of the form $u_{i}=$ $F_{i} y_{i}, \quad i \in \mathcal{I}$ to this system yields the equation $\dot{x}=H x$ where $H=A+\sum_{i \in \mathcal{I}} B_{i} F_{i} C_{i}$. For given $p \in \mathcal{I}$, explicit necessary and sufficient conditions under which there exist $F_{i}$ which make $\left(C_{p}, H, B_{p}\right)$ controllable and observable are given in [12] and [8]. There are two conditions. First, (14) must be jointly controllable and jointly observable. Second, each "complementary subsystem" of (14) must be "complete." $\{\mathrm{cf}$. Theorem 3, [8] $\}$ There are as many complementary subsystems of (14) as there are strictly proper subsets of $\mathcal{I}$. By the complementary subsystem of (14) corresponding to a nonempty proper subset $\mathcal{C} \subset \mathcal{I}$, is meant a subsystem with input matrix $\mathbf{B}(\mathcal{C})=$ block $\operatorname{row}\left\{B_{i}: i \in \mathcal{C}\right\}$, state matrix $A$ and readout matrix $\mathbf{C}(\overline{\mathcal{C}})=$ block column $\left\{C_{i}: i \in \overline{\mathcal{C}}\right\}$ where $\overline{\mathcal{C}}$ is the complement of $\mathcal{C}$ in $\mathcal{I}$ [8]. The complementary subsystem determined by $\mathcal{C}$ is uniquely determined up to the orderings of the block rows and block columns of $\mathbf{B}(\mathcal{C})$ and $\mathbf{C}(\overline{\mathcal{C}})$ respectively; as will become clear in a moment, the properties which characterize completeness do not depend on these orderings.

For a given complementary subsystem $(\mathbf{C}(\overline{\mathcal{C}}), A, \mathbf{B}(\mathcal{C}))$ to be complete, its transfer matrix $\mathbf{C}(\overline{\mathcal{C}})(s I-A)^{-1} \mathbf{B}(\mathcal{C})$ must be nonzero and the matrix pencil

$$
\pi(\mathcal{C})=\left[\begin{array}{cc}
\lambda I-A & \mathbf{B}(\mathcal{C}) \\
\mathbf{C}(\overline{\mathcal{C}}) & 0
\end{array}\right]
$$

\footnotetext{
1 The symbols used in this section such as $x, C_{i}, A, \mathcal{I}$ are generic and do not have the same meanings as the same symbols do when used elsewhere in the paper.
}

must have rank no less than $n$ for all real and complex $\lambda$ $\{$ See [13] or Corollary 4 of [12]\}. The requirement that the transfer matrix of each complementary subsystem be nonzero, can be established in terms of the connectivity of the "graph" of (14). By the graph of (14), written $\mathbb{G}$, is meant that $k$-vertex directed graph with labels in $\mathcal{I}$ and arcs defined so that there is an arc from vertex $j$ to $i$ if $C_{i}(s I-A)^{-1} B_{j} \neq 0$ for all labels $i, j \in \mathcal{I}$. For the transfer matrices of all complementary subsystems of (14) to be nonzero, it is necessary and sufficient that $\mathbb{G}$ be a strongly connected graph $\{$ Lemma 8, [8] $\}$.

\section{ANALYSIS}

The aim of this section is to prove Proposition 1 . To do this it is useful to first establish certain properties of the sub-system of 12, 13) defined by the equations

$$
\begin{aligned}
\dot{\epsilon} & =\tilde{A} \epsilon+\sum_{i \in \mathbf{m}} \sum_{j \in \overline{\mathcal{N}}_{i}} B_{i} u_{i j} \\
y_{i j} & =C_{i j} \epsilon, \quad i j \in \mathcal{J}
\end{aligned}
$$

where $\mathcal{J}$ is the complement of the set $\{i i: i \in \mathbf{m}\}$ in $\mathcal{I}$ and for $i \in \mathbf{m}, \overline{\mathcal{N}}_{i}$ is the complement of the set $\{i\}$ in $\mathcal{N}_{i}$. This subsystem is what results when outputs $y_{i i}, i \in \mathbf{m}$, are deleted from (13). Our goal here is to show that with suitable scalars $f_{i j}$, the matrix pairs $\left(\bar{H}, B_{p}\right), p \in \mathbf{m}$, are all controllable with controllability index $m$ where

$$
\bar{H}=\tilde{A}+\sum_{i \in \mathbf{m}} \sum_{j \in \overline{\mathcal{N}}_{i}} B_{i} F_{i j} C_{i j}
$$

and $F_{i j}=f_{i j} I_{n}$. Note that for any $f_{i j}$ and any $p \in \mathbf{m}$ the submatrix $\left[\begin{array}{llll}B_{p} & \bar{H} B_{p} & \cdots & \bar{H}^{m-1} B_{p}\end{array}\right]$ has exactly $n m$ columns. Since $n m$ is the dimension of the system (16), (17), $m$ is the smallest possible controllability index which the pair $\left(\bar{H}, B_{p}\right)$ might attain as the $f_{i j}$ range over all possible values. From this it is obvious that if for each $p \in \mathbf{m}$, there exist $f_{i j}$ for which $\left(\bar{H}, B_{p}\right)$ has controllability index $m$, then there must be $f_{i j}$ for which $\left(\bar{H}, B_{p}\right)$ has controllability index $m$ for all $p \in \mathbf{m}$, and moreover the set of $f_{i j}$ for which this is true is the complement of a proper algebraic set in the linear space in which the vector of $f_{i j}$ takes values.

To proceed we will first show that with the $f_{i j}$ chosen properly, the matrix pair $\left(F, b_{m}\right)$ is controllable, where $F$ is the $m \times m$ matrix

$$
F=\sum_{i \in \mathbf{m}} \sum_{j \in \overline{\mathcal{N}}_{i}} b_{i} f_{i j} c_{i j}
$$

and for $i \in \mathbf{m}, b_{i}$ is the $i$ th unit vector in $\mathbb{R}^{m}$. Note that $F$ is what results when the feedback laws $v_{i j}=f_{i j} w_{i j}$ are applied to the system

$$
\begin{aligned}
\dot{z} & =\sum_{i \in \mathbf{m}} \sum_{j \in \overline{\mathcal{N}}_{i}} b_{i} v_{i j} \\
w_{i j} & =c_{i j} z, \quad i j \in \mathcal{J}
\end{aligned}
$$

where as before, $c_{i j}$ is the row in the transpose of the incidence matrix of $\mathbb{N}$ corresponding to the arc from $j$ to $i$. Note that (20), (21) can be viewed as a $m^{*}$ channel system where $m^{*}$ is the number of labels in $\mathcal{J}$. In view of the fact that $\operatorname{span}\left\{b_{1}, b_{2}, \ldots, b_{m}\right\}=\mathbb{R}^{m}$, it is obvious that 20) is jointly 
controllable. Let $\mathbb{G}$ denote that $m^{*}$-vertex directed graph with vertex labels in $\mathcal{J}$ and arcs defined so that there is an arc from vertex $i j$ to $k q$ if $c_{k q}(s I)^{-1} b_{i} \neq 0$ for $j \in \overline{\mathcal{N}}_{i}$.

Lemma 1: If the neighbor graph $\mathbb{N}$ is strongly connected, then $\mathbb{G}$ is strongly connected.

Proof of Lemma 1; Note that for each $j \in \overline{\mathcal{N}}_{i}, c_{i j}(s)^{-1} b_{i}=$ $-\frac{1}{s}$ and $c_{i j}(s)^{-1} b_{j}=\frac{1}{s}$. From these expressions it follows that $c_{i j}(s I)^{-1} b_{i} \neq 0$ and $c_{i j}(s I)^{-1} b_{j} \neq 0$ for $i \in \mathbf{m}, j \in$ $\overline{\mathcal{N}}_{i}$. Therefore for each $i \in \mathbf{m}$, the subgraph $\mathbb{G}_{i}$ induced by vertices $i j, j \in \overline{\mathcal{N}}_{i}$ is complete. By the quotient graph of $\mathbb{G}$, written $\mathbb{Q}$, is meant that directed graph with $m$ vertices labeled $1,2, \ldots, m$ and an arc from $i$ to $k$ if there is an arc in $\mathbb{G}$ from a vertex in the set $\left\{i j: j \in \overline{\mathcal{N}}_{i}\right\}$ to a vertex in the set $\left\{k q: q \in \overline{\mathcal{N}}_{k}\right\}$. Because each of the subgraphs $\mathbb{G}_{i}$ is complete, $\mathbb{G}$ will be strongly connected if $\mathbb{Q}$ is strongly connected. But $\mathbb{Q}=\mathbb{N}$ so $\mathbb{Q}$ is strongly connected. Therefore $\mathbb{G}$ is strongly connected.

Lemma 2: If the neighbor graph $\mathbb{N}$ is strongly connected, then each complementary subsystem of (20) (21) is complete.

Proof of Lemma 2: Let $\mathcal{C} \subset \mathcal{J}$ be a nonempty subset and let $\left(\mathbf{C}, 0_{m \times m}, \mathbf{B}\right)$ be the coefficient matrices of the complementary subsystem determined by $\mathcal{C}$. Thus $\mathbf{B}=\operatorname{block} \operatorname{row}\left\{b_{i}\right.$ : $i j \in \mathcal{C}\}$, and $\mathbf{C}=$ block column $\left\{c_{i j}: i j \in \overline{\mathcal{C}}\right\}$ where $\overline{\mathcal{C}}$ is the complement of $\mathcal{C}$ in $\mathcal{J}$. To prove the lemma, it is enough to show that the coefficient matrix triple $\left(\mathbf{C}, 0_{m \times m}, \mathbf{B}\right)$ is complete. To establish completeness the transfer matrix $\mathbf{C}(s I)^{-1} \mathbf{B}$ must be nonzero and the matrix pencil

$$
\pi(\mathcal{C})=\left[\begin{array}{cc}
\lambda I & \mathbf{B} \\
\mathbf{C} & 0
\end{array}\right]
$$

must have rank no less than $m$ for all real and complex $\lambda$ $\{$ cf, Corollary 4, [12] $\}$. In view of Lemma 1 and the assumption that $\mathbb{N}$ is strongly connected, $\mathbb{G}$ is strongly connected. Therefore by Lemma 8 of [8], $\mathbf{C}(s I)^{-1} \mathbf{B} \neq 0$.

To complete the proof it is enough to show that for all complex numbers $\lambda$, rank $\pi(\mathcal{C}) \geq m$. In view of the structure of $\pi(\mathcal{C})$ in 22 , it is clear that for all such $\lambda, \operatorname{rank} \pi(\mathcal{C}) \geq$ rank $\mathbf{C}+\operatorname{rank} \mathbf{B}$. To establish completeness, it is therefore sufficient to show that

$$
\operatorname{rank} \mathbf{C}+\operatorname{rank} \mathbf{B} \geq m
$$

Let $q \in \mathbf{m}$ denote the number of distinct integers $i$ such that $i j \in \mathcal{C}$. In view of the definition of $\mathbf{B}, \operatorname{rank} \mathbf{B}=q$. If $q=m$, rank $\mathbf{B}=m$ and (23) holds. Suppose next that $q<m$. Let $\mathbf{C}^{*}$ denote the submatrix of $\mathbf{C}$ which results when all rows $c_{i j}$ in $\mathbf{C}$ for which $i k \in \mathcal{C}$ for some $k$, are deleted. Since rank $\mathbf{C} \geq \operatorname{rank} \mathbf{C}^{*}$ and $\operatorname{rank} B=q$, (23) will hold if

$$
\operatorname{rank} \mathbf{C}^{*} \geq(m-q)
$$

Corresponding to the definition of $\mathbf{C}^{*}$, let $\mathbb{N}^{*}$ denote the spanning subgraph of $\mathbb{N}$ which results when any arc in $\mathbb{N}$ from $i$ to $j$ for which there is a $k$ such that $i k \in \mathcal{C}$ is removed. There are exactly $q$ distinct values of $i$ for which $i k \in \mathcal{C}$ for some $k$. Moreover, for any such $i$ the corresponding vertex in $\mathbb{N}^{*}$ cannot have any outgoing arcs. Since $\mathbb{N}$ is strongly connected, any other vertex $k$ in $\mathbb{N}^{*}$ must have at least one outgoing arc not incident on vertex $k$. This means that the un-oriented version of $\mathbb{N}^{*}$ must have at most $q$ connected components. Thus if $M_{\mathbb{N}^{*}}$ is the incidence matrix of $\mathbb{N}^{*}$, then as a consequence of Theorem 8.3.1 of [14],

$$
\operatorname{rank} M_{\mathbb{N}^{*}} \geq m-q
$$

But for any $i j \in \mathcal{J}$ such that $i k \notin \mathcal{C}$ for some $k, c_{i j}$ is the row in the transpose of the incidence matrix of $\mathbb{N}^{*}$ corresponding to the arc from $j$ to $i$. Therefore, up to a possible re-ordering of rows, $\mathbf{C}^{*}=M_{\mathbb{N}^{*}}^{\prime}$. From this and (25) it follows that (24) holds. Therefore the lemma is true.

Lemma 3: Let $A_{n \times n}, F_{m \times m}$ and $g_{m \times 1}$ be any given realvalues matrices. There is a $m n \times m n$ nonsingular matrix $T$ such that

$$
\left[G H G \cdots H^{m-1} G\right]=\left[g \otimes I_{n}(F g) \otimes I_{n} \cdots\left(F^{m-1} g\right) \otimes I_{n}\right] T
$$

where $G=g \otimes I_{n}$ and $H=I_{m} \otimes A+F \otimes I_{n}$.

Proof of Lemma 3; Since $\left(I_{m} \otimes A\right)\left(F \otimes I_{n}\right)=\left(F \otimes I_{n}\right)\left(I_{n} \otimes\right.$ $A)$, for $k \geq 1$

$$
\begin{aligned}
H^{k} & =\left(I_{m} \otimes A+F \otimes I_{n}\right)^{k} \\
& =\sum_{i=0}^{k}\left(\begin{array}{l}
k \\
i
\end{array}\right) F^{i} \otimes A^{k-i}
\end{aligned}
$$

where $\left(\begin{array}{c}k \\ i\end{array}\right)$ is the binomial coefficient. Thus

$$
\begin{aligned}
H^{k} G & =\left(I_{m} \otimes A+F \otimes I_{n}\right)^{k}\left(g \otimes I_{n}\right) \\
& =\sum_{i=0}^{k}\left(\begin{array}{l}
k \\
i
\end{array}\right) F^{i} g \otimes A^{k-i}, \quad k \geq 1
\end{aligned}
$$

Define $T_{1}=I_{m n}$ and for $k \in\{2,3, \ldots, m\}$ let $T_{k}$ be that $m n \times m n$ matrix composed of $m^{2} n \times n$ submatrices $T_{i j}(k)$ defined so that $T_{i i}(k)=I_{n}, i \in \mathbf{m}, T_{(i+1),(k)}(k)=$ $\left(\begin{array}{c}k-1 \\ i\end{array}\right) A^{k-i-1}, \quad i \in\{0,1, \ldots, k-1\}$, and all remaining $T_{i j}(k)=0$.

Let $X(k)=\left[g \otimes I_{n} \cdots\left(F^{k-1} g\right) \otimes I_{n} H^{k} G \cdots H^{m-1} G\right]$ for $k \in \mathbf{m}$. Obviously, $X(1)=\left[\begin{array}{llll}G & H G & \cdots & H^{m-1} G\end{array}\right]$, and $X(m)=\left[\begin{array}{llll}g \otimes I_{n} & (F g) \otimes I_{n} & \cdots & \left(F^{m-1} g\right) \otimes I_{n}\end{array}\right]$.

The definition of $T_{k}$ and (27) imply that

$$
X(k) T_{k}=X(k-1), k \geq 1 .
$$

We claim that $T \triangleq T_{m} T_{m-1} \cdots T_{1}$ has the required properties. Note first that each of the $T_{i}$ is an upper triangular matrix with ones on the main diagonal. Thus each $T_{i}$ is nonsingular which implies that $T$ is nonsingular. According to (28)

$$
\begin{aligned}
{\left[g \otimes I_{n}(F g) \otimes I_{n} \quad \cdots\right.} & \left.\left(F^{m-1} g\right) \otimes I_{n}\right] T \\
= & X(m) T_{m} T_{m-1} \cdots T_{1} \\
= & X(m-1) T_{m-1} T_{m-2} \cdots T_{1} \\
& \vdots \\
= & X(1) T_{1} .
\end{aligned}
$$

Since $T_{1}=I_{m n}$, 26 is true.

Lemma 4: Suppose $\mathbb{N}$ is strongly connected. The $m^{*}+m$ channel system (12), (13) is jointly controllable and jointly observable.

Proof of Lemma 4; In view of the definitions of the $B_{i}$, it is clear that $\mathcal{B}_{1}+\mathcal{B}_{2}+\cdots \mathcal{B}_{m}=\mathbb{R}^{n m}$ where $\mathcal{B}_{i}$ is 
the column span of $B_{i}$. It follows at once that (12), (13) is jointly controllable. To establish joint observability it is enough to show that 0 is the only vector $x \in \mathbb{R}^{n m}$ for which $C_{i j} x=0, i j \in \mathcal{I}$ and $\tilde{A} x=\lambda x$ for some complex number $\lambda$. Suppose $\tilde{A} x=\lambda x$ in which case $A x_{i}=\lambda x_{i}$ where $x=\left[\begin{array}{llll}x_{1}^{\prime} & x_{2}^{\prime} & \cdots & x_{m}^{\prime}\end{array}\right]^{\prime}$ and $x_{i} \in \mathbb{R}^{n}, i \in \mathbf{m}$. Moreover, if $C_{i j} x=0, i j \in \mathcal{I}$, then $C_{i} x_{i}=0, i \in \mathbf{m}$ and $M_{I} x=0$ where $M_{I}$ is the transpose of the incidence matrix of $\mathbb{N}$. Since $\mathbb{N}$ is strongly connected, $M_{I} x=0$ implies that $x_{i}=x_{1}, i \in \mathbf{m}$. Thus $C_{i} x_{1}=0, i \in \mathbf{m}$. But $(C, A)$ is observable by assumption where $C=\left[\begin{array}{llll}C_{1}^{\prime} & C_{2}^{\prime} & \cdots & C_{m}^{\prime}\end{array}\right]^{\prime}$. Therefore $x_{1}=0$. This implies that $x=0$ and thus that (12), (13) is jointly observable.

Proof of Proposition 1; Since span $\left\{b_{1}, b_{2}, \ldots, b_{m}\right\}=\mathbb{R}^{m}$, the subsystem defined by (20) (21) is jointly controllable. From this, Lemma 2 and Theorem 1 of [8] it follows that for each $p \in \mathbf{m}$, there exist $f_{i j}$ such that $\left(F, b_{p}\right)$ is a controllable pair where $F$ is as defined (19). Since the set of $f_{i j}$ for which this is true, is the complement of a proper algebraic set in the space in which the $f_{i j}$ takes values, there also exist $f_{i j}$ for which $\left(F, b_{p}\right)$ is a controllable pair for all $p \in \mathbf{m}$. Fix such a set of $f_{i j}$.

By definition $B_{i}=b_{i} \otimes I_{n} \quad i \in \mathbf{m}, C_{i j}=c_{i j} \otimes$ $I_{n}, \quad i j \in \mathcal{J}$ and $\tilde{A}=I_{m} \otimes A$. In view of the definition of $\bar{H}$ in (18), $\bar{H}=I_{m} \otimes A+F \otimes I_{n}$. From this and Lemma 3 it follows that for each $p \in \mathbf{m}$ there is a nonsingular matrix $T_{p}$ such that $\left[\begin{array}{lllll}B_{p} & \bar{H} B_{p} & \cdots & \bar{H}^{m-1} B_{p}\end{array}\right]=$ $\left(\left[\begin{array}{llll}b_{p} & F b_{p} & \cdots & F^{m-1} b_{p}\end{array}\right] \otimes I_{n}\right) T_{p}$. Since each $T_{p}$ is nonsingular and each $\left(F, b_{p}\right)$ is a controllable pair,

$$
\operatorname{rank}\left[\begin{array}{llll}
B_{p} & \bar{H} B_{p} & \cdots & \bar{H}^{m-1} B_{p}
\end{array}\right]=n m
$$

Therefore for each $p \in \mathbf{m},\left(\bar{H}, B_{p}\right)$ is a controllable pair with controllability index $m$. Note that if we define $F_{i i}=0, i \in \mathbf{m}$, then in view of (11), $H=\bar{H}$. Therefore, for each $p \in \mathbf{m}$, $\left(H, B_{p}\right)$ is a controllable pair with controllability index $m$. Clearly this must be true generically, for almost all $F_{i j}, i j \in \mathcal{I}$.

In view of Theorem 1 of [8], the complementary subsystems of (12) and (13) must all be complete. But by Lemma 4, (12) and (13) is a jointly controllable, jointly observable system. From this and Corollary 1 of [8], it follows that there exist $F_{i j}, i j \in \mathcal{I}$ such that for all $p \in \mathbf{m}$ and all $q \in \mathcal{N}_{p}$, the matrix pairs $\left(H, B_{p}\right)$ and $\left(C_{p q}, H\right)$ controllable and observable respectively. Since this also must be true generically for almost all $F_{i j}$ the proposition is true.

\section{CONCLUDING REMARKS}

In this paper we have explained how to construct a family of distributed observers for a given neighbor graph $\mathbb{N}$ which are capable of estimating the state of the system (11) (2) at an preassigned but arbitrarily fast convergence rate. There are many additional issues to be addressed. For example, how might one construct distributed observers of least dimension which can estimate $x$ ? Accomplishing this will almost certainly require the transmission to each agent $i$ from each neighbors $j$, the signal $y_{j}$ which agent $j$ measures. This of course comes at a price, so there is a trade-off to be studied between required observer dimension on the one hand and the amount of information to be transferred across the network on the other. Another issue of importance would be to try to construct a distributed observer for the case when $\mathbb{N}$ changes over time; of course this problem will call for a different type of mathematics since the equations involved will be time-varying systems. Finally it would be useful to try to determine how to construct distributed observers when in place of (2), one has $\dot{x}=A x+\sum_{i=1}^{m} B_{i} u_{i}$ where $u_{i}$ is an input signal which can be measured by agent $i$. Some of these problems will be addressed in the future.

\section{ACKNOWLEDGEMENT}

The authors wish to thank Shinkyu Park and Nuno C. Martins for useful discussions which have contributed to this work.

\section{REFERENCES}

[1] S. Park and N. C. Martins. Design of distributed lti observers for state omniscience. IEEE Transactions on Automatic Control, 2017. to appear

[2] R. Olfati-Reza and J. S. Shamma. Consensus filters for sensor networks and distributed sensor fusion. In Proc IEEE CDC, page 512 520, 2005.

[3] R. Carli, F. Fagnani, A. Speranzon, and S. Zampieri. Communication constraints in the average consensus problem. Automatica, pages 671684, 2008.

[4] L. Xiao, S. Boyd, and S. Lall. A scheme for robust distributed sensor fusion based on average consensus. In Proc. Fourth International Symposium on Information Processiing in Sensor Networks, pages 63$70,2005$.

[5] R. Olfati-Reza. Kalman-consensus filter: Optimality, stability, and performance. In Proc IEEE CDC, pages 7036-7042, 2009.

[6] F. Dorfler, F. Pasqualetti, and F. Bullo. Continuous-time distributed observers with discrete communication. IEEE Journal of Selected Topics in Signal Processing, 7(2):296-304, 2013.

[7] S. H. Wang and E. J. Davison. On the stabilization of decentralized control systems. IEEE Transactions on Automatic Control, 173. 473478.

[8] J. P. Corfmat and A. S. Morse. Decentralized control of linear multivariable systems. Automatica, 12(5):479-497, September 1976.

[9] D. G. Luenberger. Observing the state of a linear system. IEEE Transactions on Military Electronics, pages 74-80, 1964.

[10] W. M. Wonham. Dynamic observers - geometric theory. IEEE Transactions on Automatic Control, pages 258-259, 1970.

[11] F. M. Brasch and J. B. Pearson. Pole placement using dynamic compensators. IEEE Transactions on Automatic Control, pages 34-43, 1970.

[12] J. P. Corfmat and A. S. Morse. Control of linear systems through specified input channels. SIAM Journal on Control, 14:163-175, 1976.

[13] J. M. Potter, B. D. O. Anderson, and A. S. Morse. Single-channel control of a two-channel system. IEEE Transactions on Automatic Control, 24:491-492, June 1979.

[14] C. Godsil and G. Royle. Algebraic Graph Theory. Springer, 2001.

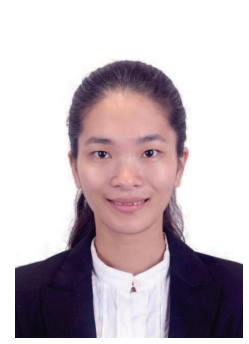

Lili Wang received the B.E. and M.S. degrees from Zhejiang University, Zhejiang, China, in 2011 and 2014, respectively. She is currently a Ph.D. student majored in electrical engineering in the School of Engineering \& Applied Science, Yale University, USA. Her research is on the topic of cooperative multi-agent systems and distributed observer. 


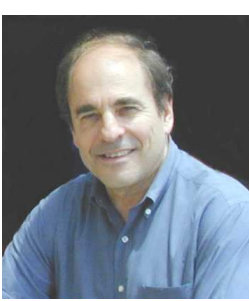

A Stephen Morse received a Ph.D. degree from Purdue University. Since 1970 he has been with Yale University where he is presently the Dudley Professor of Engineering. He has received several awards including the 1999 IEEE Technical Field Award for Control Systems and the American Automatic Control Council's 2013 Richard E. Bellman Control Heritage Award. He is a member of the National Academy of Engineering. 